\title{
Evaluar lo oral ${ }^{\star}$
}

\author{
Claudine Garcia-Debenc \\ Claudine GARCIA- DEBENC. \\ IUFM de Toulouse, INRP.**
}

Las directrices institucionales, especialmente las propuestas por Ségolène Royal para el colegio a partir del año 2000, ${ }^{1}$ insisten en la importancia del aprendizaje de lo oral. Estas buscan, a partir de ese momento, legitimar su enseñanza por parte de los maestros y de los inspectores. Lo anterior incluye la escuela primaria, el colegio, la enseñanza profesional y el liceo. Elizabeth Nonnon ha señalado que antes de esta disposición solamente la escuela maternal y la educación especializada se habían interesado en la didáctica de lo oral. Aunque lingüistas especializados, como Claire Blanche Benveniste, prefieran utilizar el término de "francés hablado", ${ }^{2}$ yo retomo el término de "oral" 3 para conservar la referencia a la trilogía aceptada por el Ministerio de Educación Nacional Francés actual para referirse a "el dominio de las lenguas": "escrito - oral - imagen".

Si lo oral llega a ser un conjunto de objetos de enseñanza como cualquier otro implica que se defina de manera operatoria y precisa su evaluación. Ahora bien, uno todavía encuentra, aunque con poca frecuencia, la rúbrica "oral" en los boletines escolares del segundo grado o en las libretas de competencias para el primero. Cuando se agrega una competencia, no sé si esto sea positivo puesto que los maestros la describen a menudo de forma muy subjetiva y bajo juicios globales como por ejemplo "participa-no participa", los cuales son poco representativos de las verdaderas competencias discursivas de los niños. Este fenómeno no es exclusivo de Francia, ya nuestra colega de Ginebra Béatrice Berset Fougerand había subrayado las dificultades ocasionadas por la entrada en vigencia de un "nuevo boletín escolar que hacía explícitamente de la expresión oral uno de los componentes de la evaluación del francés Ilamado de expresión", lo cual era válido en 1988. En ese momento los profesores de Ginebra señalaban sus dificultades para proceder a una evaluación de lo oral.

* Traducción de Pilar Mestre de Caro, Universidad de La Salle, y Raquel Pinilla Vásquez, Universidad Distrital Francisco José de Caldas. ** Garcia-Debenc, C. (1999). « Evaluer l'oral ». Revista Pratiques, 103-104. IUFM de Toulouse, INRP. Francia. Texto traducido con autorización de la autora y de la editora de la revista.

1 Le Collège des années (2000).

2 Para una presentación clara y sintética de las características sintácticas del francés oral, se podrá leer Blanche-Benveniste (1997). 3 Con el fin de guardar cierta fidelidad con el texto original, conservaremos a lo largo del artículo el término "oral" para referirnos a la modalidad oral del lenguaje y al uso oral de la lengua. 
La reflexión que nos proponemos realizar en este documento es sobre "la evaluación de lo oral" con todas las comillas necesarias tanto alrededor del término "evaluación" como del término "oral". Para ello es necesario un triple punto devista.

Primero que todo, para lo oral como para los otros dominios de la enseñanza, las herramientas de evaluación son la parte visible del iceberg constituido por el zócalo de competencias que son el objeto de una enseñanza y de un aprendizaje. Dime que evalúas y te diré que enseñas. El estudio de los instrumentos de evaluación constituye entonces un buen soporte para analizar los contenidos de enseñanza y las competencias esperadas.

Comenzar por la evaluación parece igualmente muy pertinente desde un punto de vista epistemológico. La reflexión sobre los instrumentos de evaluación y las teorías de referencia actuales sobre lo oral, ta como será desarrollado en la tercera parte de este artí culo, permite indagar por las concepciones subyacentes de lo oral y su pertinencia científica. El trabajo que aquí propongo pretende contribuir un poco a evitar las desviaciones tecnicistas que correrían el riesgo de producir la concepción de instrumento de evaluación sin tener en cuenta de manera suficiente las especificidades lingüísticas del francés hablado. Hoy está de moda hablar de lo oral y producir rápidamente materiales destinados a los docentes la demanda del mercado es fuerte- sin que haya realmente suficiente familiaridad con el tratamiento de la lengua hablada por parte de los diseñadores de manuales o de materiales de enseñanza. De ahí los riesgos que se corren de un retorno a la norma que niega las especificidades de lo oral y de una desnaturalización completa de su enseñanza.

Para finalizar, un trabajo parecido es indispensable en la formación de docentes con el fin de hacer emerger y cuestionar las representaciones sobre el francés hablado y lo que puede y debe constituir el objeto de enseñanza. De esta manera en los cursos de for- mación continuada destinada a los docentes de colegio así como en un módulo dirigido a los Profesores de Escuelas de segundo año de IUFM, propuse un grupo de diez instrumentos de evaluación más o menos discutibles y les pedí a los docentes, por grupos alrededor de cinco, que escogieran y eliminaran uno de esos instrumentos, justificando sus elecciones; podían enseguida adaptar uno de estos instrumentos proponiendo una modalidad de empleo en su institución. Algunos de estos fueron escogidos y otros rechazados por grupos diferentes, lo que permitió, en el curso de una puesta en común, confrontar las representaciones sobre la enseñanza de lo oral y sobre la norma.

\section{Problemas específicos que plantea la evaluación de lo oral 1.1 ¿Por qué es tan difícil evaluar lo oral?}

Aunque está continuamente presente en todas las prácticas de la clase, lo oral no es a menudo reconocido por lo docentes como objeto de enseñanza y su evaluación es juzgada por ellos como difícil, e incluso "peligrosa". Esto está sin duda relacionado con diez razones tanto de orden empírico como teórico.

1. La práctica de lo oral es transversal a todas las disciplinas y a todas las situaciones, de tal manera que son difícilmente aislables de los objetos de enseñanza susceptibles de ser trabajados. Lo oral está por todas partes en la escuela, fuera de ella, en la clase y en el recreo. La comunicación oral se considera espontánea. Su desarrollo se efectúa antes de la entrada a la escuela, en gran parte de manera empírica y no consciente. Por ello es difícil de hacer de lo oral un "objeto de aprendizaje" como si se puede hacer con otras materias más específicas en el mundo escolar, en particular todos los aprendizajes que se relacionan con lo escrito, incluso si estos también se construyen en parte fuera de la escuela. Esto proporciona de otra parte a los docentes la excusa de no trabajar lo oral puesto que se practica permanentemente... 
2. Lo oral es difícil de observar y complejo de analizar. Los parámetros que intervienen en la interpretación de un enunciado oral son numerosos y concomitantes: a los elementos sintácticos y semánticos, hay que agregarles especialmente la entonación, la prosodia, las variaciones de cadencia, las pausas ${ }^{4}$... Estos parámetros que pueden ser decisivos para la interpretación de un enunciado, son difíciles de describir si no se cuenta con la ayuda de instrumentos tecnológicos complicados.

3. Lo oral implica el conjunto de la persona. La producción verbal no puede ser disociada de la voz y del cuerpo. Todo significa: espacio, distancias sociales (tal como se estudian en la proxémica ${ }^{5}$ ), hábitos culturales y los silencios que tampoco pueden serignorados. A menudo se deduce de ellos un sentimiento de inseguridad, de suerte que los aspectos psicológicos y afectivos son en ocasiones decisivos, mucho más que en las producciones escritas. ¿Cómo evaluar una competencia si no es partir de los desempeños? Y ¿cómo evaluar las competencias orales de un alumno que nunca toma la palabra? Nuestro colega canadiense Jean-Marie Doutrelouxhabía puesto en evidencia, hace ya muchos años, el carácter multireferenciado de una evaluación de lo oral.

Es más, muchos profesores y ciertos alumnos son reticentes a re escuchar su voz o a volver a ver su imagen: no se pueden negar los problemas psicológicos y deontológicos de la imagen de sí que nunca deja de poner problemas en un trabajo como este. De otra parte, las grabaciones a menudo tienen una incidencia importante sobre las producciones verbales obtenidas: ciertos alumnos tímidos no se arriesgan a hablar, otras personas quieren hablar todo el tiempo sin que verdaderamente tengan algo que decir. Los profesores en general tienen más reticencias a dejarse filmar que a suministrar documentos escritos sobre sus prácticas de enseñanza.

No es fácil saber cómo evaluar en el momento una interacción oral en su complejidad y con sus implicaciones afectivas. Es tal vez por esto que los principales instrumentos de evaluación que se pueden encontrar se relacionan con situaciones de "presentaciones orales" como la recitación o la lectura oral, que no comprometen el conjunto de la persona y sífocalizan la atención sobre la cadencia, la articulación o los movimientos de la mirada. En el inventario de prácticas de enseñanza de lo oral que dieron lugar a una evaluación en dos escuelas primarias, Dominique Bétrix Kohler y AnneMarie Piguet analizaron situaciones de lectura en voz alta, de juego dramático, de sainete teatral y de grabación de un comentario para un montaje audiovisual. Esta última situación, aunque utiliza el medio oral, presenta también muchas analogías con lo escrito, puesto que uno puede borrar, recomenzar y re escucharse cómo se haría en lo escrito.

4. Desde el punto de vista sociolingüístico, lo oral está profundamente marcado, más que en lo escrito, por las prácticas sociales de referencia ${ }^{6}$. Esta problemática, bien conocida en sociolingüística y en la enseñanza del Francés como Lengua Extranjera, es a menudo ignorada por los docentes especialmente los de $Z_{E P}{ }^{7}$ (Zona de Educación Prioritaria) quienes sienten como agresivas las variaciones de intensidad (los alumnos hablan fuerte), de cadencia (hablan rápido) de distancia proxémica (hablan muy cerca), aspectos

4 Para una descripción sintética de estos fenómenos para el francés, se podrá consultar Morel y Danon-Boileau (1998).

5 "Es posible pensar que de acuerdo con los tipos de interacción, los locutores adoptarán distancias inter-individuales y posiciones diferentes, acordes con las reglas culturales que aunque generalmente son implícitas, no son por lo tanto menos precisas" (Cosnier, 1978). Edward Hall precisó diversos tipos de distancias adaptadas para un adulto culto norteamericano, distinguiendo entre la distancia íntima, la distancia personal, la distancia social y la pública (de 1,20 m a 3,60 m) para compararlas con las de otras poblaciones como las árabes y latinoamericanas. 6 Sobre esta pregunta, Consultar Langage et Société (1997) de la colección Mémo Seuil, obra redactada por Josiane Boutet. La editorial Seuil propone una síntesis muy clara y accesible.

7 Las ZEP están destinadas a abordar problemas relacionados con los índices de fracaso y deserción escolar. Se encuentran ubicadas en lugares o zonas socialmente desfavorecidas. Fueron propuestas en 1981 y desde su creación han sido objeto de evaluaciones y de reformas (Nota de las traductoras). 
que revelan esencialmente variaciones culturales. Es indiscutible la importancia de difundir conocimientos sobre estos aspectos en la formación de docentes, por ello es importante preguntarse por el interés de una objetivación y de un análisis de estas variaciones en el espacio de la clase: metacomunicar sobre las diferencias entre prácticas familiares y prácticas escolares, en otras palabras, ¿es legitimo y aconsejable reconsiderar las prácticas familiares y compararlas con las escolares? Esto es en todo caso muy difícil de poner en práctica.

5. Lo oral no deja huellas y necesita para su estudio de grabaciones técnicamente exigentes. Las producciones orales no pueden ser seriamente analizadas sino pueden ser re escuchadas muchas veces. De esta manera, mientras que uno puede cómodamente leer y releer un escrito, un trabajo oral necesita grabaciones de audio y de video, transcripciones y re escuchas, lo que demanda material técnico de grabaciones $y$ mucho tiempo, además puede eventualmente causar problemas deontológicos y jurídicos con los padres de los alumnos cuando se procede a realizar grabaciones en las clases. Halliday consideraba, en 1985, que la invención de la grabadora portátil debería ser considerada como una fecha determinante para el desarrollo de la lingüística de lo oral. La grabadora es también a la vez una herramienta para la investigación y una herramienta pedagógica. Catherine Decout, profesora de liceo y quien trabaja regularmente lo oral en clase propone a sus alumnos de decimo grado, en el marco de la preparación para la prueba oral del bachillerato, archivar sus exposiciones en un casete para que después puedan escucharse nuevamente. De la misma manera, la enseñanza a distancia realiza la corrección de exposiciones. En investigación, el video permite identificar más fácilmente los locutores.
Por otra parte, surge la pregunta sobre qué lugar darle a un trabajo metalingüístico en la enseñanza de lo oral: ¿es necesario que los alumnos vuelvan a escuchar y a mirar sus actuaciones? La didáctica de la producción escrita se ha fundado, en los últimos veinte años, en la realización regular de actividades de evaluación formativa de los escritos y de la re escritura. Algunos maestros o investigadores en didáctica ${ }^{8}$ han trasladado estos métodos para lo oral sin interrogarse verdaderamente sobre las especificidades de la producción oral. De esta manera proponen momentos de escucha y de análisis de emisiones televisivas con el fin de que los alumnos deduzcan criterios de buena actuación, re escuchas de actuaciones de los alumnos así como elaboración de borradores sucesivos. Pero uno puede preguntarse si la producción verbal oral y la escrita son de la misma naturaleza. La escritura tiene precisamente como especificidad permitir un distanciamiento (se puede escribir yo sin ser yo, como lo muestran los casos de distanciamiento entre narrador y autor, lo que es muy difícil en lo oral, salvo en una escena de teatro), una objetivación, un volver atrás en el curso de producción y una transformación sin dejar rastros. ¿Es esto igual para lo oral, que tiene como característica ser lineal y sucesivo? Lo dicho, dicho está como dice el proverbio popular. En lo oral, yo no puedo borrar y, para rectificar una afirmación, estoy obligado a agregar (Él vino ayer. Finalmente no, tal vez antes de ayer), el espacio de dos dimensiones de lo escrito permite borrar y reemplazar. Desde este punto de vista, se pueden comparar los tachones que reemplazan un elemento por otro con las añadiduras sucesivas y necesarias en lo oral para rectificar o ajustar una formulación. Si desde la perspectiva de un aprendizaje sistemático de lo oral, retomar las producciones verbales para analizarlas con los alumnos parece necesario, este trabajo no podría operar de manera idéntica para lo oral que para lo escrito. En una didáctica de lo oral, más que la "re escritura" de lo oral, imposible por definición, es necesario con-

8 Pienso, por ejemplo, en nuestros colegas y amigos de Ginebra, Bernard Schneuwly y Joaquim Dolz (1998), cuya obra ha tenido una gran acogida entre los docentes en Francia. 
cebir un conjunto de situaciones que presenten una homología suficiente para que los alumnos puedan reconocerlas, identificarlas como familiares y transferir sus competencias en curso de construcción, pero al mismo tiempo suficientemente variadas para que ellos sientan ganas de tomar la palabra.

6. La evaluación de lo oral requiere mucho tiempo. Otro obstáculo para la evaluación de lo oral es el tiempo que exige, tanto en el espacio de la clase como en el trabajo personal del docente.

En el espacio de la clase, los medios disponibles en el colegio y en el liceo solo permiten una evaluación muy ocasional de los desempeños de cada uno de los estudiantes. Es más, los docentes tienen la idea de que solamente con la "participación" los alumnos tendrán logros en lo oral. Ahora bien, el análisis detallado de las intervenciones en clase en un "curso dialogado" muestra que, en este caso, los alumnos no producen sino fragmentos que se insertan en el habla magistral. De tal manera que el diálogo no es más que un monólogo a muchas voces. Si la evaluación opera a partir de exposiciones, presentaciones de libros en clase, debates, el número de alumnos que pueden ser seriamente evaluados en cada curso es, en este caso insuficiente y se puede, en este caso, tener miedo de pasar más tiempo en evaluar lo oral que en enseñarlo. Por consiguiente un trabajo serio, solo puede ser conducido en grupos pequeños con clases organizadas en módulos de tal manera que lo oral no monopolice los tiempos de trabajo.

El otro obstáculo se relaciona probablemente con el anterior. Lo maestros pueden llevar a sus casas las copias y corregirlas. El tiempo de trabajo personal que ellos consagran a la evaluación de lo escrito es importante en la vida de los profesores. En cambio no está bien visto si algunos de ellos llevan a sus casas casetes de audio o de video para corregirlos. Hay posibilidades de que esto empeore sus condiciones de trabajo ${ }^{9} \mathrm{Y}$ se sabe que los profesores no aceptan una innovación si la ganancia no les parece superior al costo.

Evaluar lo oral no debería de otra parte significar evaluar exclusivamente la producción verbal. Así como en lo escrito se evalúa la escritura y la lectura, en lo oral se debería evaluar más la escucha. En el marco de la investigación del INRP "Lo oral para aprender", yo había solicitado individualmente a los alumnos de CM1 (equivalente al cuarto grado de básica primaria) reformular lo que se acababa de decir en el curso de una sesión de ciencias consagrada a la confrontación de representaciones escritas de los alumnos a propósito de la respiración. Esto me permitió constatar que ciertos alumnos "participan" mucho pero son incapaces de reformular lo que dicen sus compañeros y que otros alumnos, mudos en el curso de la interacción, han integrado perfectamente todos los objetivos. En la evaluación oral, es importante no limitarse a tomar en cuenta exclusivamente la producción verbal, como se hace generalmente, sino concebir también una evaluación y un aprendizaje de la escucha.

7. La evaluación de lo oral supone un paso por lo escrito por la vía de las transcripciones. El análisis de las dimensiones sintáctica y semántica de lo oral solo es posible recurriendo a las trascripciones escritas. Estas pueden tomar diferentes formas en función de los objetivos perseguidos, desde trascripciones fonéticas hasta trascripciones ortográficas. Estas necesitan ser escuchadas varias veces para trascribir exactamente las superposiciones y no proyectar sobre la trascripción segmentaciones propias de la frase escrita, que no recubren sistemáticamente los sistemas de pausas de lo oral. 
Desde el punto de vista de la enseñanza oral en clase y de la formación de docentes, uno puede preguntarse si vale la pena trascribir los corpus orales para los alumnos, para los docentes de formación inicial y en formación continua y para qué les puede servir. A partir de los años 70, en las Escuelas Normales los formadores de docentes procedían regularmente a la transcripción de grabaciones orales para poner en evidencia las diferencias de funcionamiento entre la lengua oral y la lengua escrita. La hegemonía de la lingüística aplicada condujo a menudo a realizar un trabajo similar con los alumnos. Tales actividades permiten tomar conciencia de la diferencia de segmentación entre lo oral y lo escrito (en lo oral, no hay segmentación por palabras ni por unidades sintácticas), de las variaciones sintácticas $\mathrm{y}$, con los alumnos, permite tomar conciencia de las especificidades de las estructuras sintácticas en lo escrito. De esta manera, por ejemplo, como lo han mostrado los trabajos de Anne Claude Berthoud, hay maneras de trabajar un tema muy diferente en lo oral de lo que se puede hacer en lo escrito. Un trabajo así tiene como ventaja mejorar el conocimiento de lo oral en los docentes y de evitar los juicios normativos o reductores.

8. Lo oral es a menudo mal conocido, su funcionamiento especifico ignorado. Se juzga siempre la calidad de lo oral a partir de lo escrito, desvalorizando siempre lo oral. Así sea en los diccionarios o en los juicios sobre las producciones, la afirmación "es propio de lo oral" corresponde siempre a un juicio negativo. Denise François, en el numero 26 de Pratiques denunciaba las transgresiones paródicas de una falsa oralidad en los discursos políticos de los oradores de comienzo de siglo.

Existen variaciones en lo oral más importantes aún que en lo escrito. ¿Cuáles son los puntos comunes entre el discurso escrito oralizado del discurso político y la conversación informal? Las dos producciones difieren profundamente tanto desde el punto de vista estrictamente lingüístico como por el lugar que ocupan en la interacción en cada uno de estos casos. La interacción oral se caracteriza por el trabajo interlocutivo permanente sobre los objetos de discurso como lo describe Elisabeth Nonnon. Esta cogestión permanente de objetos de discurso difiere notablemente de lo oral, que Lahire llama escritural , y que él presenta como factor determinante del éxito escolar.

9. Los indicadores de dominio de lo oral no son claramente sintetizados. ¿Qué es dominar lo oral para un alumno de final de la escuela primaria? de colegio? de liceo? Mientras que existen para lo escrito indicadores de la construcción progresiva de las competencias, estos no son conocidos para lo oral. Cuando existen, estos indicadores se relacionan especialmente con la escuela maternal. Para los otros niveles, aparte de criterios relativos a la entonación, a la cadencia y a la gestión de elementos no verbales de la comunicación (mirada...), se dan pocas indicaciones precisas. No obstante Elisabeth Nonnon se esfuerza por elucidar estos indicadores a través de un análisis fino de los turnos de habla en una conversación en grupos. Ella detecta las competencias en materia de gestión de las interacciones, cuando un alumno muestra que es capaz de "darle ritmo al intercambio haciendo bucles recapitulativos, operando él mismo los desniveles conclusivos y sacando mayor ventaja de lo que se propone".

10. Hasta hace poco el material pedagógico para enseñar lo oral era escaso y recientemente se han desarrollado más las investigaciones didácticas sobre lo oral que sobre lo escrito. Lo oral se lleva difícilmente a manuales y fichas, aunque existen inventarios de ejercicios, especialmente en la formación de adultos. Ciertos manuales del ciclo 3 han comenzado a proponer una rúbrica consagrada a lo oral en la cual proponen actividades, que son a menudo artificiales y simplistas teniendo en cuenta la complejidad y la variedad de situaciones de lo oral. 
El hecho de que los maestros dispongan de poco material didáctico para organizar un trabajo sobre lo oral con sus alumnos, explica también el carácter reciente de las investigaciones al respecto. En los años setenta, el Plan de Renovación de la Enseñanza del Francés ya había destacado la importancia del trabajo de lo oral y sus propósitos para hacer retroceder el fracaso escolar (Brunner, Fabre y Kerloc'h, 1985). Este trabajo estaba orientado hacia una perspectiva de liberación de la expresión, pues otorgaba un lugar importante a las lenguas regionales. Contrariamente a las perspectivas actuales, trataba al aprendizaje de lo oral en autonomía e incluso en concurrencia con lo escrito. Actualmente las investigaciones sobre didácticas de lo oral comienzan a desarrollase de una parte en Suiza en la Facultad de Ciencias de la Educación de Ginebra, al rededor de Bernard Schneuwly; por otra parte, en Francia, especialmente bajo la dirección del INRP (investigación: "Lo oral para aprender") y del Centro de Investigaciones en Didáctica del Francés de Metz. Más adelante desarrollaremos los puntos en los que se oponen estas investigaciones

\subsection{Seis buenas razones para evaluar lo oral}

1. Que sea o no enseñado, lo oral es evaluado, especialmente en los exámenes o en las entrevistas de trabajo. De suerte que los objetivos sociales del dominio de lo oral son inmensos. En efecto, la competencia oral es a menudo esencial en los exámenes (como el "gran oral" de la Escuela Nacional de Administración (ENA). Es más, las producciones orales convocan juicios sociales permanentes y a menudo inconscientes que implican hábitos culturales. La fluidez de lo oral es muy variable según los medios sociales. Si se considera que el sistema escolar tiene por misión favorecer el éxito escolar y social en una perspectiva de democratización, un trabajo sobre esta dimensión es necesario desde la escuela primaria, pero también en el colegio y en el liceo. Aun si, por imitación o por ósmosis, ciertos alumnos de algunos medios sociales se apropian fácilmente de las reglas de lo oral escolar, una evaluación explícita puede contribuir a un mejor éxito para los alumnos de rendimiento mediano.

2. Toda evaluación de lo oral implica una reflexión sobre la norma (¿qué es hablar bien?). Como lo hemos dicho, la apreciación de la lengua "hablada" se asocia a menudo con juicios peyorativos. Ahora bien, la competencia comunicativa consiste en adaptar su discurso a la situación y no en "hablar como un libro". ¿Cuál oralidad se busca enseñar? Esto lo veremos más adelante en la tercera parte de este artículo.

3. El estatus de lo oral en la clase es un buen analizador del modo de trabajo pedagógico y de la concepción de aprendizaje del maestro. El volumen relativo de la palabra del maestro y de los alumnos, el carácter más o menos cerrado de las preguntas hechas por el docente, el lugar reservado a las solicitudes de explicitación o de justificación, la naturaleza y el estatus de las reformulaciones, son los índices más importantes de la relación de lo oral con los aprendizajes tal como se presenta en el espacio de la clase.

4. Una evaluación objetiva es necesaria para que los docentes puedan proceder a un apoyo eficiente. El concepto de andamiaje es tomado de Bruner: "hay andamiaje explícito cuando un adulto ayuda al niño a hacer o a decir eso que él no podría hacer solo" (Frédéric, 1993). Ahora bien, como lo señala Elisabeth Nonnon (1997, p. 35), para proceder a un andamiaje eficaz y adaptar sus intervenciones en el transcurso de la interacción misma, el docente " tiene la necesidad de leer en los enunciados orales de los alumnos todas sus conductas en gestación, aun bajo forma embrionaria, como es a menudo el caso, cuando las "estrategias prometedoras" complejizan el desarrollo de la investigación y de la puesta en palabras en detrimento de la corrección y de la simplicidad de los enunciados. Es el caso los alumnos de CM1 (cuarto grado de básica primaria), cuando argumentan a propósito de las cualidades de un libro. En lugar de argumentos en el sentido canónico del término ("aprecio un libro cu- 
ando es chistoso"), ellos se ponen contar episodios de la novela, que no escogidos al azar, de tal manera que él es bueno porque esperado por el docente se transforma en es bueno cuando (Garcia-Debenc, 1996, pp. 50-66). Una evaluación fina de lo oral es por tanto necesaria para organizar lo que Linda Allal denomina una regulación retroactiva (1988).

5. Los docentes necesitan una evaluación objetiva para programar eficazmente una enseñanza de lo oral. Una evaluación precisa es igualmente necesaria para organizar una regulación proactiva (25), es decir para realizar por adelantado actividades que permitan construir un cierto número de competencias orales. Bétrix Köhler y Anne-Marie Piguet (1991) observan que, en el marco de proyectos que implican lo oral,

[...] los maestros involucrados regulan más a menudo las actividades con el fin de conducirlas bien, de asegurar su éxito (un espectáculo para los padres, por ejemplo) en vez de preocuparse por ayudar a los alumnos a gestionar ciertos aprendizajes previstos para el dominio de lo oral. La dificultad para definir los objetivos de aprendizaje a alcanzar durante una actividad participa ciertamente de la explicación de este fenómeno (Betrix Köhler y Piguet, 1991, p. 178).

$\mathrm{Si}$, tradicionalmente, se programan las actividades de lectura o de enseñanza de la lengua, esto raras veces se hace para lo oral.

6. Una evaluación objetiva es necesaria a los alumnos para saber cómo progresar. Si una evaluación precisa y objetiva es indispensable para los maestros, ella es igualmente útil a los alumnos para saber cómo progresar. De ahí el interés de formular periódicamente con ellos criterios de evaluación de lo oral en función de las diferentes situaciones de producción.

Como "hay que trabajar lo oral", los maestros se esfuerzan en hacerlo, sin que necesariamente todos pongan la misma cosa bajo esta rúbrica. Antes de interrogarse sobre las modalidades y los criterios de evaluación de lo oral, debe interrogarse primero que todo sobre el estatus de la enseñanza de lo oral en la clase.

\section{2. ¿Cuál objeto de enseñanza? Razones principales}

\section{1. ¿Evaluar actividades autónomas 0 actividades integradas?}

Dos vías posibles se ofrecen al docente que busca trabajar explícitamente lo oral con sus alumnos: la que propone el equipo de Bernard Schneuwly, en Suiza, que consiste en trabajar los géneros de lo oral, por ejemplo el debate público, la entrevista radiofónica, la lectura en voz alta, bajo la forma de módulos de entre nueve a doce sesiones autónomas que contengan sesiones de producción oral y también de escucha y de análisis de grabaciones, de producción escrita de notas de trabajo y de lectura. El estudio de cada género puede organizarse en un conjunto de horas específicas consagradas a lo oral, por ejemplo dividiendo la clase. Esta organización didáctica tiene como ventajas la legibilidad de los trabajos sobre lo oral y un tecnicismo en su enfoque. Corre el peligro de atomizar aún más el trabajo en clase de lengua creando una nueva subdisciplina, que aunque está en interacción con la lectura y la producción de escritos, es autónoma, sin que esto cuestione el estatus de la interacción oral en las demás sesiones de lengua.

La otra vía que han escogido los equipos de primer grado del INRP (por ejemplo los artículos que están en este mismo número de la revista de G. Turco y de S. Plane por el INRP y el conjunto de los que están consagrados al "Corpus de Adverbio" por el CRDF ), quienes prefieren realizar una mirada particular a las interacciones cotidianas en clase. Los equipos del INRP intentan, para cada una de las sesiones, explicitar los objetivos y los indicadores de éxito que corresponden al doble punto de vista de los aprendizajes disciplinares y de las conductas discursivas desarrolladas. De esta manera, para la aproximación de la argumentación oral en sexto grado, más que organizar los debates sobre temáticas al alcance de 
los alumnos de este grado, pero sin un propósito real (¿cuál animal doméstico escoger?, ¿es mejor ir a una colonia en vacaciones o quedarse en casa?, o como lo propone un material de enseñanza bajo la forma de una denominada "situación lúdica": “¿matamoscas o bomba insecticida?"), el docente puede escoger trabajar la argumentación oral a partir de libros que los niños pueden recomendar o no a sus compañeros, o a partir de contradicciones entre las interpretaciones de lectura de los niños, por ejemplo sobre el número de personajes en escena al comienzo de una novela. En este caso, los aprendizajes en juego son claramente aprendizajes de lengua, pero las situaciones puestas en juego tienden más a suscitar justificaciones y refutaciones por parte de los alumnos. Por ejemplo, en un juego de roles, en parejas, dos alumnos voluntarios van respectivamente a recomendar o no la lectura de una novela a sus compañeros quienes indicarán si el debate los hizo cambiar de punto de vista. Esta discusión puede, según el caso, ser la ocasión de una reflexión más general sobre los criterios para la selección de libros o de una aclaración sobre la significación profunda de una ficción, como lo hemos visto en una clase del CM (ciclo medio) a propósito de la novela de Marguerite Yourcenar Cómo fue salvado Wang-Fô (Garcia-Debenc, 1996, pp. 50-66).

\section{2.¿Cuál(es) práctica(s) social(es) de referencia?}

Las elecciones necesarias para poner en marcha una didáctica de lo oral no pueden economizar la reflexión sobre las prácticas sociales de referencia. La selección de estas prácticas que fundamentan la enseñanza de lo oral se deriva del punto precedente o posiblemente le son subyacentes. Cuando los profesores deciden hacer trabajar a los estudiantes sobre una entrevista radiofónica o un debate televisado, Bernard Schneuwly y Joaquim Dolz inscriben el trabajo escolar en consonancia con los usos sociales de lo oral en los medios de comunicación. Ellos califican estos géneros como "formas", "en la medida que son producidos en las instituciones de la vida pública" (Enjeux 1997, p. 88). Su elección se inscribe en un movimiento paralelo a aquel que escoge como objetos de enseñanza para la producción escrita textos funcionales análogos a los que son más utilizados en la sociedad, como la carta, las reglas de juego o el aviso clasificado. Las modalidades de trabajo son completamente análogas: igual que en una interacción lectura-escritura bien conducida, a los alumnos se les pide observar los rasgos específicos de diversos géneros de escritos sociales. En una didáctica de lo oral así concebida, a los alumnos se les conduce a escuchar y a analizar extractos de emisiones radiofónicas o televisivas que constituyan un corpus de textos como fuentes de referencia. Sin embargo, las finalidades de un trabajo como este son muy diferentes de aquellos que serían asignados a una formación profesional de periodista: aquí se trata de iniciar un debate televisivo, no para formar profesionalmente a periodistas, sino para educar al ciudadano telespectador en la escucha y observación de procedimientos. Una elección como esta permite localizar los objetos de enseñanza y hacer trabajar a los alumnos sistemáticamente, con el riesgo de no modificar las condiciones de interacción en la clase.

Una opción diferente puede hacerse al otorgarle un lugar propio a la comunicación escolar en su especificidad. Esta adquiere así un estatus de práctica social de referencia específica. Aunque existe un gran número de trabajos teóricos que tratan las interacciones orales desde una perspectiva del análisis conversacional, pocos estudios han sido consagrados hasta el momento a la especificidad de las interacciones en situación escolar. Se sabe que es en la interacción escolar y desde la escuela maternal que se ponen en juego los mecanismos de éxito o de fracaso escolar y que, además, la escuela es la instancia social principal de producción de normas. No cabe duda de que es en las interacciones orales, tal como ellas se desarrollan en las aulas, que se construyen en los alumnos las representaciones de lo que la es- 
cuela admite como lengua legítima. Es la elección que hace el CRDF (en esta publicación) al tratar la función didáctica de las interacciones, como lo hace el INRP en sus investigaciones sobre "lo oral para aprender".

\section{3. ¿Interacción oral u oral escritural?}

¿Cuál oral se busca enseñar ? Lo oral de la exposición presenta muchas características comunes con lo escrito, tanto desde el punto de vista de sus condiciones de producción y de planificación, como desde el punto de vista de sus características sintácticas. Quien hace una exposición debe, como si escribiera, decidir solo las explicaciones que va a dar, organizar su habla de manera individual, prever las incomprensiones posibles de su auditorio... para hacerlo, a menudo le es muy útil que redacte por escrito su comunicación, al menos a grandes rasgos. En el curso de la producción verbal, su atención se focaliza sobre la producción de frases completas muy próximas a la sintaxis de lo escrito. En este sentido, la oralidad de la exposición se diferencia notablemente de la oralidad conversacional, tal como se inscribe en una interacción social.

Al analizar las prácticas discursivas orales de la escuela primaria, Bernard Lahire (1993) constata que "la escuela es el lugar en donde se trata la palabra de los alumnos como la combinación de una sintaxis y de un vocabulario". De hecho, el examen de las prácticas escolares muestra que "las prácticas discursivas orales solo toman su sentido cuando se las relaciona con las formas sociales escritas, es decir que las formas de relaciones sociales han sido históricamente posibles por la práctica de la escritura, los saberes escriturales y la relación con el lenguaje y el mundo que les es indisociable". Esta relación con el lenguaje es común a las prácticas que le reclaman a la escuela y a las prácticas de las familias más fuertemente dotadas escolarmente; esto explica las dificultades escolares de los niños provenientes de clases sociales que tienen una relación más "práctica" con la lengua oral. Elisabeth Bautier y Jean-Yves Rocheix han mostrado las incidencias de esta explicación sociológica sobre la relación con el saber de los nuevos estudiantes de liceo (1998). Para favorecer el éxito escolar de los alumnos habitualmente excluidos del sistema educativo, es necesario trabajar con ellos la diversidad de usos orales, poniéndolos en la posición de practicar en la escuela a la vez la práctica oral conversacional que les es familiar y lo oral escriptural que privilegia la escuela. Por esto es interesante introducir situaciones orales autónomas en las cuales los estudiantes puedan explicar solos o de a dos, o de a tres, un fenómeno científico que han entendido de otros compañeros, ${ }^{11}$ situaciones en las cuales lo oral es trabajado a la vez en su dimensión escrita y con su características de lo oral. Para cada una de las situaciones de lo oral, es importante que el docente sea claro sobre el tipo oral que él trabaja.

\subsection{Sintaxis de lo oral y la norma}

Los modos de producción específicos de la lengua hablada explican ciertas características sintácticas de lo oral, como la abundancia de dislocaciones ("mi padre, él llega"), construcciones fragmentadas ("lo que yo puedo decir es que es una buena idea"), reiteraciones ("hay tres prácticas tres semanas de prácticas"). Igualmente, en francés oral es normal la ausencia de la primera parte de la negación ne salvo en ciertos contextos. Como lo muestra Claire Blanche-Benveniste, estos rasgos característicos de las formas de lo oral más conversacional son a menudo juzgados como "errores" y son descalificados.

De la misma manera, en el aula, el docente tiene a menudo la costumbre de "normar en exceso" su habla o la de los alumnos exigiéndoles, en francés, la doble negación o estructuras subordinadas en expresiones orales en donde éstas no son naturales. La contradicción era particularmente sensible en el marco de los

11 Como en la situación descrita y analizada por Michel Grandaty en la revista Repères, 17. 
ejercicios estructuralistas en boga en los años setenta, que realizaban entrenamientos de lo oral a partir de la producción de estructuras de frases precisamente poco naturales en lo oral y características de lo escrito. A falta de conocimiento suficiente de las características sintácticas del francés hablado, el docente corre el riesgo de hacer practicar a sus alumnos un oral imposible, en lugar de apoyarse en las prácticas espontáneas de lo oral para hacer observar las diferencias entre estas estructuras sintácticas y las que deben ser utilizadas en lo escrito en situaciones orales más formales, sin inducir a la existencia de una jerarquía entre estas diversas formas de práctica discursiva.

\subsection{Inventario de variables didácticas como ayuda para la selección de una programación}

Lo oral puede tomar formas de realización muy diferentes según las situaciones de comunicación en las cuales se produce, de manera que, como acabamos de verlo, no hay un oral sino diversos tipos de oral . La investigación del INRP "Oral II" está en curso de elaborar un inventario provisional de variables didácticas. Esto consiste en exponer las diversas dimensiones que deben tenerse en cuenta para organizar una enseñanza de lo oral. Busca ayudar a los docentes a regular su enseñanza de lo oral tomando conciencia de las elecciones que son necesarias, de las dimensiones que privilegian en su enseñanza y de las que ellos descartan deliberadamente o por ignorancia. El repertorio de estos diversos lugares de variación puede ayudar a programar actividades diversas. A continuación un inventario provisional:

\section{Naturaleza de la actividad oral}

- Participación en una interacción/oral monogestionada/oral escritural/escrito oralizado.

\section{Lugar de la actividad en el proyecto de aula}

- Campo disciplinar/"oral ciudadano" (participación en la gestión del aula o de la escuela).
- Actividad integrada a un proyecto/provocada/simulación (juego de rol).

- Grado de ritualización: actividad excepcional/actividad frecuente. Actividad ritualizada.

\section{Modalidades de organización del grupo-clase}

- Organización del grupo: grupo-clase/trabajo en grupos (tamaño del grupo, constitución de los grupos por afinidad o según sus competencias...).

- Presencia/ausencia del maestro en el momento de la producción oral.

- Naturaleza de los interlocutores: al interior de la clase, entre las clases.

- Roles explícitos asignados a los alumnos (portavoz, instructor, regulador)/ausencia de roles.

- Disposición espacial.

\section{Prácticas sociales de referencia}

- Género con existencia social (entrevista, debate)/ género escolar (exposición)/interacción ordinaria en clase.

- Toma en cuenta de registros de radio o televisión.

- Registro de lengua seleccionado.

\section{Naturaleza de las conductas discursivas esperadas}

- Objetivo discursivo: explicar, convencer...

- Conducta discursiva: narrar, describir, explicar, argumentar.

- Monogestionada/poligestionada.

\section{Tareas discursivas}

- Grado de precisión.

- Naturaleza de la consigna.

\section{Relación escrito/oral}

- Relación con lo escrito: no escrito/escrito para leer/ para producir. 
- Naturaleza de lo escrito: esquema/tabla/notas/ texto.

- Momento en el que interviene lo escrito: antes de lo oral/entre dos fases orales/después de lo oral.

\section{Naturaleza del andamiaje por parte del docente}

- Naturaleza de las preguntas: abiertas/cerradas.

- Naturaleza y frecuencia de las reformulaciones.

- Nivel de tratamiento didáctico privilegiado: gestión de turnos de habla/discursivo/lexical/sintáctico/ fonológico/prosódico/no verbal.

\section{Lugar y forma de un análisis metalingüístico}

- Utilización de herramientas de grabación: video/ audio/uso de un micrófono.

- Momento: pausa en curso de acción/análisis en vivo/observación o escuchas sucesivas en grupos/ observación o escuchas sucesivas en grupo-clase.

- Elaboración de instrumentos de evaluación.

- Modalidades de elaboración de instrumentos de evaluación: interacción oral/redacción en grupos de indicadores para observar/redacción individual de indicadores para observar o de observación.

- Utilización de instrumentos de evaluación.

- Modalidades de utilización de instrumentos de evaluación.

\section{Para recordar, las variables independientes son las siguientes}

- Prácticas sociales de referencia de los alumnos. Especialmente zonas de educación prioritaria (ZEP)/ no ZEP; medio social de origen; estilos educativos familiares...

- Estilo pedagógico del docente (concepción de aprendizaje, modalidades de tratamiento del error, lugar acordado para las socializaciones...).

Cada una de las situaciones de lo oral desarrolladas en una clase selecciona ciertos parámetros. La puesta en práctica de una didáctica de lo oral coherente supone que, en un año escolar o en un ciclo, sean practicadas situaciones de oralidad variadas que hayan permitido a los alumnos explorar las diferentes dimensiones de las actividades orales.

\section{Análisis crítico de una muestra de instrumentos de evaluación para la escuela primaria y secundaria}

Desde un punto de vista lingüístico, el desconocimiento generalizado del funcionamiento sintáctico y prosódico de lo oral (tal como lo describieron por ejemplo Claire-Blanche Benveniste, 1990, 1997; o Marie-Annick Morel, 1998) explica tal vez las grandes ingenuidades que se pueden constatar en algunos instrumentos de evaluación. Para evaluar lo oral, es necesario incluso preguntarse qué concepción se tiene de lo oral y definir algunas de sus características lingüísticas.

Los creadores de materiales para la enseñanza de lo oral o los investigadores en didáctica difunden una concepción particular de lo oral, a través de los instrumentos de evaluación que proponen. De manera indirecta, estos instrumentos de evaluación constituyen entonces, en su escala, un medio de formación sobre lo oral para los docentes. Autores como Le Cunff y Jourdain (1999) tienen razón al denunciar la mediocre calidad general de los instrumentos para la evaluación de lo oral: "Estas rejillas están a menudo incompletas o demasiado cargadas, son poco fiables o limitantes, cuando no son todo esto a la vez" (p. 187). Esta observación, que puede aplicarse también a los instrumentos de evaluación formativa de las producciones escritas, se aplica aún más al empleo de herramientas de evaluación de lo oral, en la que no siempre es posible hacer varias lecturas sucesivas.

Desde un punto de vista epistemológico, es interesante interrogar las concepciones subyacentes de lo oral. Lo haremos aquí a partir de siete proposiciones de instrumentos de evaluación dirigidos a públicos diferentes, desde la escuela primaria hasta el bachil- 
lerato. Para cada uno de estos instrumentos, pondremos en evidencia en el análisis un aspecto sobresaliente, que aparecerá en el título del párrafo.

\subsection{Un instrumento para la escuela primaria sin indicador preciso de competencia lingüística o la ilusión de la participación}

El primer instrumento de evaluación analizado aquí es extraído de la obra reciente del Grupo Oral-Créteil, coordinado por Le Cunff y Jourdain, Enseñar lo oral en la escuela primaria, París, Hachette Education. El capítulo consagrado a la evaluación se interesa por las condiciones necesarias para establecer una pedagogía diferenciada: "se trata de determinar entre los alumnos, cuáles son los que se expresan delante de toda la clase sin que se les pida, y cuáles son aquellos que se expresan solamente en grupos pequeños, incluso únicamente cara a cara. La observación se hace entonces en el plano de la cantidad de intervenciones al momento de los regrupamientos, con la clase completa". Estos principios están inspirados por la noción de grande y pequeño orador desarrollada por Agnès Florin (1995). La rejilla de observación del equipo Oral Créteil, presentada en la página 189, intitulada "rejilla sobre los desempeños lingüísticos", distingue efectivamente tres situaciones de comunicación (grupo grande/grupo pequeño/cara a cara) y dos modalidades de intervención (habla espontánea/habla solicitada). Los tres criterios de evaluación, aplicados de manera binaria están codificados por una letra:

- S: significa "dentro del tema/fuera del tema".

- M: significa "toma la palabra en buen momento", de buen/mal grado.

- F: significa "se expresa en las formas esperadas/no" (las comillas están ubicadas en el mismo lugar que en el texto original).

Un instrumento de este tipo contiene la representación de los docentes sobre el hecho de que es al hablar que los niños aprenden a hablar, y que entre ellos más "participan", más progresos realizan. Formulado de esta manera, dicha herramienta permite tal vez medir el grado de inhibición de un niño en un grupo de clase, pero en ningún caso da cuenta, ni de lejos ni de cerca, de la naturaleza de sus desempeños lingüísticos orales, como lo anuncia el título. Los criterios de evaluación propuestos a los docentes dejan abierta la posibilidad a la subjetividad y a lo arbitrario. Los dos primeros, codificados con $\mathrm{S}$ y $\mathrm{M}$, se refieren a aspectos relativos a la gestión de los turnos de habla; están formulados de manera tan general que se vuelven esencialmente comportamentales. En cuanto al tercero, "se expresa de la forma esperada/no" aun si, además, está indicado que las "formas esperadas" corresponden a características de conductas discursivas diferenciadas señala una relación a la norma de manera muy maniqueista, lo que da lugar a la subjetividad de los docentes.

El lector espera con impaciencia la respuesta a la pregunta formulada en el último párrafo: “¿Qué criterios para evaluar y definir objetivos?". Cito a continuación la integralidad de este párrafo que parece incompleto: "Los criterios de evaluación son necesarios y se construyen a partir de las instrucciones oficiales, en la medida en que estas son explícitas. Sin embargo, como lo indica el párrafo anterior, es posible apoyarse en los logros de los alumnos de "buen desempeño" para construir un "conjunto de indicadores" en lo que respecta al resto de los alumnos, a condición de relacionar los desempeños esperados con las categorías que constituyen los componentes de las tomas de palabra" (estas han sido referidas en los párrafos precedentes: aspectos lingüísticos, principalmente léxico y sintaxis, competencias metalingüísticas, trabajo sobre sí mismo).

Tal herramienta de evaluación, demasiado vaga para poder ser realmente operativa para los docentes, deja una parte muy importante a la apreciación subjetiva. Además, se fundamenta de manera esencial sobre criterios 
de orden comportamental, subestimando la dimensión estrictamente lingüística; no tiene en cuenta las especificidades lingüísticas del francés hablado.

El segundo instrumento considerado es tomado de un folleto editado por el CRDP de Alsacia y el IUFM de Alsacia, titulado ¡Para que cada uno hable!, consagrado más particularmente al desarrollo de las competencias orales en la escuela materna y en CP (curso preparatorio). Este propone una tentativa de caracterización de los desempeños a alcanzar y se organiza en cuatro rúbricas:

- Aspectos "físicos" de la lengua oral (toma de la palabra, pronunciación, ubicación de la voz, postura corporal).

- Aspectos socializantes de la lengua oral (inteligncia del objetivo y dimensión relacional relativa a los turnos de habla).

- Aspectos del dominio lingüístico de lo oral.

- Aspectos funcionales de la lengua que reagrupan la enumeración de diversas conductas lingüísticas (relatar-narrar, describir, argumentar...).

- Es en la tercera rúbrica, relativa al dominio lingüístico de lo oral, en la que nos interesaremos particularmente aquí. Esta se divide a su vez en cuatro puntos:

- Adaptación del tiempo de conjugación/temporalidad de la acción.

- Construcción sintáctica: a) simple, luego b) compleja.

- Adecuación del léxico utilizado/pertinencia de la idea expresada.

- Desarrollo de una/varias ideas en un discurso (varias frases coherentes encadenadas: tema-palabras).

Esta enumeración de criterios está acompañada por una nota: "Código común a lo escrito/oral, sabiendo que desde la escuela maternal, algunos niños producen frases complejas de manera espontánea por haberlas aprendido con adultos".

Algunos criterios están formulados de manera muy general, por lo cual no se ve bien lo que los docentes pueden hacer realmente: esto sucede con el empleo de los tiempos verbales o con la utilización del léxico. ¿Cómojuzgar la calidad de un desempeño oral cuando no se da ninguna indicación precisa sobre los indicadores objetivos que se deben identificar para juzgar el nivel de competencia de un niño de esta edad? Así mismo, para el encadenamiento tema-palabras, la formulación no tiene en cuenta la especificidad de los encadenamientos a nivel oral y a nivel escrito (Berthout, 1996 ). Los elementos más debatibles teóricamente se refieren a los aspectos sintácticos: los puntos a) y b) y el conector luego permiten pensar que las construcciones simples son anteriores y más fáciles de dominar que las construcciones Ilamadas complejas.

¿Se entiende por esto el número de lo que anteriormente Laurence Lentin llamaba "introductores de complejidad"? El principio parece presentado aquí como si el número de introductores de complejidad fuera el signo de un mayor dominio, mientras que la observación de enunciados orales muestra por lo contrario que una saturación en conectores por ejemplo es, en el nivel escrito, signo de una planificación paso a paso.

Igualmente la nota deja pensar que la producción de una frase compleja es en sí misma un logro, mientras que la formulación de una frase minimalista puede ser interpretada como el signo de una competencia para diferenciar enunciación escrita y enunciación oral. En lugar de proceder a una toma de información cuantitativa, es más interesante observar el grado de variabilidad de los desempeños de un niño dado y la variedad de marcas lingüísticas que utiliza, por ejemplo para resolver el problema de la referencia, según que hable o que produzca un texto escrito.

Los autores de este material de enseñanza manifiestan así un desconocimiento del funcionamiento lingüístico de lo oral y mantienen esta confusión entre los futuros usuarios de su material. 


\subsection{Un instrumento que propone una evaluación centrada en la dimensión interaccionista para los alumnos de bachillerato}

El tercer instrumento que presentamos aquí es tomado de una contribución de Monique Maquaire en un folleto editado por el CRDP de Rennes: Hacia el dominio de los discursos, editado en la colección "Cartas abiertas" por el CRDP de Bretaña. El texto no se dirige al público presentado en el material anterior, pues se remite a las producciones orales de alumnos de bachillerato. La evaluación se limita a una competencia particular, la atención que el locutor acuerda a su interlocutor o a su auditorio. La rejilla propuesta se organiza en tres rúbricas:

- Indicadores no verbales: miradas, gestos, cadencia, articulación, modulaciones de la voz.

- Indicadores verbales: lengua.

- Relación verbal/no verbal: ¿La gestual es redundante?/¿Complementaria o en contradicción con el discurso?

Analizaremos con más detalle los ítems que aparecen en la segunda rúbrica, relativa a los indicadores verbales. Los elementos lingüísticos retenidos en esta ocasión son los siguientes:

- Indicadores de personas (pronombres de $1^{\mathrm{a}}$ y de $2^{\mathrm{a}}$ persona, on, nous, en francés; nosotros en español).

- Uso de deícticos.

- Segmentación.

- Repeticiones y redundancias.

- Énfasis.

- Dirigir la palabra al otro (¿entiende?, esto le va a causar gracia...).

- Control y adaptación del registro de lengua.

- Metadiscurso (explicaciones, comentario, empleo de fórmulas como eso quiere decir).
La evaluación es selectiva y se caracteriza por la prioridad acordada a las marcas relativas a la enunciación. El inventario preciso de las marcas lingüísticas fácilmente identificables y significativas de las reformulaciones propias de lo oral permite focalizar la observación. Contrariamente a los dos puntos precedentes, este instrumento respeta el hecho de que la atención que se le presta al otro se efectúe de manera diferente en el nivel oral y en el nivel escrito.

\subsection{Comparación de cuatro herramientas que tratan una conducta discursiva: la argumentación}

El aprendizaje de la argumentación oral presenta problema a los profesores de secundaria, especialmente en el nivel de séptimo, grado en el cual los docentes se preguntan cómo proceder a la iniciación de la argumentación con jóvenes estudiantes. Varias vías son posibles:

- De acuerdo con una tendencia que llamaré "nueva retórica", ilustrada por Dominique Brassart y el equipo de la Revista Investigaciones (Recherches) de l'AFEF de Lille (1991, 1998 ), se puede iniciar a los jóvenes del ciclo de observación a la argumentación haciéndolos debatir sobre temas acordes con su nivel, como por ejemplo "tomar el tren o el automóvil", "ir al centro vacacional o quedarse en la casa", " ¿se deben comer golosinas?", “qué mascota escoger para la familia?". Tales temas de discusión involucran a los alumnos; favorecen la toma de posiciones definidas. Al conducirlos a tener en cuenta otros puntos de vista diferentes del suyo, participan en la formación del ciudadano. Las actividades de argumentación son entonces autónomas y un poco artificiales en la medida en que no corresponden a problemas reales para los niños.

- Una segunda dirección, que calificaré como literaria, consiste en proponer a los estudiantes redactar pasajes argumentativos en los que se interesan en las obras literarias que son además estudiadas en su 
obra integral: diálogos argumentativos en una novela, monólogos en una obra de teatro... empleando los elementos dados en el contexto de la obra.

- Una tercera vía busca trabajar la argumentación como totalmente integrada a la disciplina de las letras, por medio del dominio de la justificación oral y escrita. Esta preocupación corresponde a una exigencia escolar (la justificación escrita de las respuestas es requerida en todas las disciplinas) y se constatan dificultades importantes experimentadas por los alumnos (son los ítems de este tipo los que reciben los puntajes menos favorables en la evaluación de séptimo). Esta elección evita una dicotomía entre enseñanza de la lengua y análisis de textos literarios en la medida en que la justificación de una interpretación es una primera iniciación al comentario compuesto. ¿A qué forma de discurso pertenece este escrito?, ¿cuántos personajes hay al comienzo de la novela? ¿Cómo hacer para encontrar el sujeto de un verbo? Una cantidad de preguntas que exigen justificaciones y refutaciones orales en el marco de la clase de lengua.

- La justificación puede igualmente ser trabajada en las diversas disciplinas a propósito de fenómenos científicos, que se refieren por ejemplo al medio ambiente. Estas tratan sobre "los procesos del topo" o preguntas como las siguientes: “¿Los zorros son animales perjudiciales?" o “es necesario eliminar las plagas del jardín? Los objetos sobre los cuales versa nuestra argumentación son entonces de naturaleza científica. La búsqueda de pruebas a través de situaciones de argumentación busca el mejoramiento de los conocimientos en el campo científico.

El instrumento A es tomado de la obra de Joaquim Dolz y Bernard Schneuwly ya citado. Es concebido para la evaluación del debate. Contiene siete ítems que deben ser apreciados cada uno negativa y positivamente:
1. El alumno toma la palabra.

2. El alumno da su opinión.

3. El alumno presenta diversos argumentos.

4. El alumno respeta los turnos de habla.

5. El alumno escucha a sus compañeros.

6. El alumno establece el diálogo con los otros.

7. La voz es audible para el público.

Las formulaciones son simples y muy accesibles para los maestros y para los alumnos. Los ítems 4 y 5 remiten a la gestión de los turnos de habla y a un comportamiento social en la interacción; contrariamente a los ítems 2 y 3 , que son comunes a toda situación de argumentación oral o escrita, el ítem 7 se refiere a la intensidad vocal en relación con la situación de comunicación y concierne entonces específicamente una situación de lo oral. Es lamentable que el instrumento no precise a través de cuáles marcas lingüísticas se puede apreciar que "un estudiante entra en diálogo con los otros". Para ser realmente operativo y ayudar a traspasar un juicio muy subjetivo, una herramienta de este tipo sigue siendo demasiado general. La dimensión lingüística está ausente.

El instrumento B es tomado del material publicado por las ediciones Magnard (Blochet, et ál. 1999) para el tercer ciclo de la escuela primaria. Un capítulo está consagrado a la práctica del debate. Diversas situaciones se proponen: elección entre la bicicleta todo terreno o la escalada para una tarde recreativa, diálogos en los que se organiza una lotería o el carnaval de fin de año, "debate lúdico" definido como "debate cómico a partir de hechos insignificantes", simulación de un debate televisivo sobre la creación de una autopista en el departamento. Se nota en estos ejercicios la ausencia de problemática real en los debates propuestos, de manera que el aprendizaje se limita a una dimensión retórica y formal (debatir sobre cualquier tema) en lugar de la puesta en marcha de la dimensión ciudadana y ética de las prácticas de la argumentación. Las competencias perseguidas son cuidadosamente definidas en la p. 94 pero los criterios de logro, defini- 
dos progresivamente de acuerdo con las situaciones son de una generalidad sorprendente: "respetar las reglas del juego" y "ser convincente". Nos gustaría saber qué procedimientos lingüísticos son precisamente útiles para ser convincente.

El instrumento $C$ fue perfeccionado por el equipo investigativo CERF del IUFM de Toulouse Oral Cycle 3, asociado a la investigación INRP nacional "Lo oral para aprender". El equipo de Toulouse se interesa de manera más particular en las actividades de argumentación oral integradas a las actividades de francés y en las disciplinas científicas. El instrumento $C$ fue elaborado por el grupo constituido por investigadores y docentes tras el análisis de grabaciones de video transcritas de algunos momentos de clase, en particular los momentos de socialización de actividades.

A cada uno de los criterios, indicados con negrita , corresponden diversos niveles de realización, desde un primer embrión de manifestación de competencia hasta un nivel de excelencia. El orden en el cual son formulados aquí los diversos criterios no tiene relevancia especial.

\section{Evaluación de las conductas argumentativas en lo oral Gestión del intercambio}

- Dominio de la gestión de turnos de habla (empleo pertinente de los lugares transicionales) (Sacks, Schegoff y Jefferson, 1974, pp. 696-735).

- Conservación del tema de la controversia o de la temática (no desviar).

- Escucha de la argumentación del interlocutor hasta el final (no cortar la palabra).

- Entonación que favorece la escucha de su argumento por parte de los interlocutores (ser audible, no ser agresivo).

\section{Eficacia de la gestión de la interacción}

- Encadenamiento con el discurso del otro para complementarlo.

- Adaptaciones lexicales.

- Variedad de reformulaciones.

\section{Pertinencia de las intervenciones de cada interlocutor}

- Escucha y utilización de los argumentos de un interlocutor.

- Reformulación de la intervención de otro locutor como objeto de consenso provisional.

- Reformulación del razonamiento de los otros participantes del intercambio.

- Intervención que abre el intercambio hacia una nueva controversia.

\section{Pertinencia de las justificaciones}

- Formulación de un argumento pertinente.

- Formulación de un argumento retomado luego por los otros participantes del debate.

- Presencia de un ejemplo pertinente.

- Reformulación de una característica del objeto que se va a analizar, finalizando de manera argumentativa.

\section{Toma en cuenta de las argumentaciones de los interlocutores y refutación}

- Toma en cuenta de un argumento del interlocutor.

- Empleo de un argumento del interlocutor.

- Reformulación de un argumento del interlocutor.

- Refutación de un argumento del interlocutor.

- Modificación de la orientación argumentativa de un planteamiento de un interlocutor. ${ }^{12}$ 


\section{Dominio del metalenguaje de la argumentación}

- Observaciones pertinentes y retrospectivas sobre las condiciones de éxito de una argumentación: identificación de la orientación argumentativa, evaluación de la eficacia de los argumentos, identificación de estrategias...

- Utilización pertinente del metalenguaje de la argumentación.

En esta herramienta, la noción de conducta discursiva es central. Si existen numerosos ítems comunes a lo oral y a lo escrito, la dimensión interaccionista es tenida en cuenta de manera específica.

Con su número importante de ítems, un instrumento de este tipo es demasiado pesado para ser empleado en la práctica, en la medida en que supone sucesivas escuchas de una grabación. Puede ser utilizado para analizar globalmente los desempeños de una clase, o por lo contrario, para seguir el conjunto de turnos de habla de un alumno.

El último instrumento se ha inspirado de la rejilla precedente, corresponde a la variación de la tabla de análisis $C$ realizada por una docente que pertenece al grupo de investigación con sus alumnos de CM 2 (quinto grado de básica primaria) de una clase de ZEP. Los alumnos de esta clase fueron a ver una película que no han visto los otros alumnos del colegio. Dos alumnos voluntarios, acompañados por un director de la actividad, van a tratar de convencer a los alumnos de los dos grupos CM 1 (cuarto grado de básica primaria) de la importancia de esta película: un alumno al que le gustó la película va a recomendarla, otro a desaconsejarla. La primera interacción en una de las clases fue grabada en video. Al observarla de manera colectiva se pudieron establecer criterios antes de la presentación de estos dos alumnos ante el otro curso. El dispositivo propone entonces una evaluación formativa de lo oral y una especie de reescritura. Al final de la visualización de la grabación se elaboró la herramienta siguiente.

\section{Herramientas para argumentar en forma oral}

- Sé a quién se dirige mi argumentación.

- Conozco el objetivo de mi argumentación.

- Busqué numerosos argumentos pertinentes.

- Busqué ejemplos pertinentes.

- Planeé la contraargumentación.

- Contraargumento sobre el mismo tema.

- Retomo el argumento del otro y lo cuestiono.

- Manifiesto mi acuerdo en cuanto a algunos argumentos del otro.

- Sintetizo los argumentos precedentes.

- No me salgo del tema.

- Escucho al otro sin interrumpirlo.

- Establezco los vínculos por medio de conectores.

- Miro a mi (o a mis) interlocutor(es).

La herramienta está formulada en primera persona. Sirve a la vez de guía para el alumno antes de la realización de su presentación y como instrumento de autoanálisis. Este instrumento de evaluación es artesanal: no tiene sentido sino para la clase que lo elaboró. Efectivamente, como para los otros instrumentos de evaluación formativa es el tiempo de elaboración de herramientas el que es aun más decisivo que el resultado en sí.

\subsection{Un instrumento que manifiesta un desconocimiento de la especificidad lingüística del francés hablado}

A pesar de que traten la misma conducta discursiva, la argumentación, y de que se dirijan a una misma fracción de edad, los alumnos de escuela primaria y de comienzo de secundaria, los cuatro instrumentos de evaluación que acabamos de analizar manifiestan competencias que se deben construir entre los alumnos y en el estatus acordado a la interacción. Su utilización eficaz supone una formación suficiente de los docentes en el campo de lo oral. 
¿Qué se debe hacer para formar a los docentes para evaluar y para enseñar lo oral? La labor es compleja. Nos parece en todo caso que conviene ante todo que los docentes sean sensibilizados ante la diversidad de situaciones y ante los diversos tipos de discursos orales: no son competencias idénticas las que se necesitan para participar eficazmente en una interacción en un grupo numeroso, en un grupo pequeño; para hacer una exposición oral autónoma o para decir un texto escrito. No son los mismos criterios los que permitirán apreciar los desempeños de los alumnos en estas diversas situaciones. Es por medio de la confrontación de situaciones variadas que los docentes pueden poner en evidencia, de manera progresiva, los invariantes y las variaciones y no reducir la enseñanza de lo oral a la teatralización o a la práctica de cuentos.

De manera más global, es conveniente intervenir en las representaciones generalmente descalificantes de lo oral y adoptar una reflexión profunda sobre la norma. Las variaciones son necesarias tanto a nivel escrito como a nivel oral. Todo docente debe tener claridad sobre lo que este tolera y lo que sanciona pero también cuestionarse sobre su propia producción verbal en el espacio de la clase. No debe, sin embargo, juzgar lo oral desde el marco de lo escrito: las estructuras de las frases son profundamente diferentes en estos dos tipos de expresión (oral y escrita), como lo muestra la excelente síntesis de Claire Blanche-Benveniste sobre la lengua hablada en francés.

El punto central del peritaje profesional en materia de lo oral, pero más globalmente del aprendizaje, es ser capaz de poner en marcha las condiciones para un andamiaje eficaz por parte del docente. Es lo que hemos tratado de desarrollar en el IUFM de Toulouse en el marco de un Plan Individual de Formación (PIF) opcional con practicantes PE 2, proponiéndoles elementos teóricos basados en el análisis de extractos de grabaciones de video realizadas en la clase de maestros formadores, y luego pidiéndoles que preparen en grupos pequeños momentos de socialización que ellos han conducido posteriormente en cada una de las clases, sucesivamente a propósito de un aprendizaje científico (¿qué es un freno?, ¿cuáles son las evoluciones tecnológicas de la bicicleta?, ¿por qué los automóviles fabricados en tecnología circulan o no?) y de un aprendizaje de la lengua (¿cómo hacer para encontrar el sujeto de un verbo?, ¿qué historia escoger para montar un espectáculo de teatro?, ¿qué libros pedir para una biblioteca?). Dichas actividades ponen en juego no solamente competencias discursivas, en este caso argumentativas, sino también competencias en tecnología, en física, en gramática... El andamiaje por parte del docente, en particular por medio de sus reformulaciones, debe tener constantemente en cuenta estos dos tipos de objetivos.

La formación supone también entrenarse en la definición de indicadores de logros en función del tipo de conducta discursiva trabajada (narrar, describir, explicar, argumentar), pero también en función de la edad de los niños.

Finalmente, para tomar conciencia de su propio comportamiento verbal, es igualmente útil un autoanálisis que permita identificar la parte gestual, su entonación, su fluidez... y controlarlos de manera más consciente, incluso si este trabajo es difícil y complejo.

\section{Referencias}

Allal, L. (1988). Vers un élargissement de la pédagogie de maîtrise: processus de régulation interactive, rétroactive et proactive. En Huberman, M., Assurer la réussite des apprentissages scolaires? Les propositions de la pédagogie de maîtrise. Neuchâtel et Niestlé .

Bautier, E. \& Rochex J. Y. (1998). L'expérience scolaire des nouveaux lycéens. Démocratisation ou massification. Arman Colin .

Berset Fougerand, B. (1991). Evaluer certificativement I' expression orale... ou se battre contre des moulins a vents. En Wirthner, M., Martin, D. \& Perrenoud, P ., Parole étouffée, parole libérée. Fondements et limites d'une pédagogie de l'oral (183-194). Neuchatel: Delachaux et Niestlé. 
Berthoud, A. C. (1996). Paroles à propos. Approche énonciative et interactive du topic. Paris: Ophrys.

Betrix Köhler, D. \& Piguet, A. M. (1991). Ils parlent, que peut-on évaluer? En Wirthner, M., Martin, D. \& Perrenoud, $P$., Parole étouffée, parole libérée. Fondements et limites d'une pédagogie de l'oral (171-182). Neuchatel: Delachaux et Niestlé.

Blanche-Benveniste, C. (1990). Le français parlé. Etudes grammaticales. Paris: Editions du CNRS.

- (1997). Approches de la langue parlée en français. Paris: Ophrys.

Blochet, B. et ál. (1999). Maîtriser l'oral. Cycle 3. Magnard Ecoles.

Bouchard, R. (1993). L'interaction comme moyen d'étude didactique. Interaction et processus de production écrite. Une étude de pragmatique impliquée. En Halte, J. F. (Ed.), Inter-actions (137-199). Université de Metz.

Brunner, C., Fabre, S. \& Kerloc'h J. P. (1985). Et l'oral alors?, Plan de renovación de la enseñanza del francés en la escuela primaria. Paris: Nathan.

Clermont, P., Cunin, A. \& Scheidhauer, M. L. (1997). Pour que chacun parle la l' école maternelle et au CP. Strasbourg: CRDP d'Alsace-IUFM d'Alsace .

Dolz, J. \& Schneuwly, B. (1998). Les capacités orales des apprenants. En Dolz, J. \& Schneuwly, B., Pour un enseignement de l'oral. Initiation aux genres formels à l'école (75-89). Paris: ESF.

(1998). Pour un enseignement de l'oral. Initiation aux genres formels à l'école. Paris: ESF.

Doutreloux, J. M. (1983). Prise en charge et évaluation de l'oral en primaria Ppmaf, Université de Sherbrooke.

Florin, A. (1995). Parler ensemble en maternelle. Paris: Ellipses.

François, D. (1980). Du pouvoir de l'écrit. Pratiques, (26): 25-31.

François, F. (1993). Pratiques de I'oral. Paris: Nathan Pédagogie, Théories et Pratiques.

Garcia-Debenc, C. (1996). Quand Des Élèves De Cm1 Argumentent. Langue Française, (112): 50-66.

- (1997). Pour une didactique de l'argumentation orale avec des élèves de 10 ans. Enjeux, (39/40): 50-79.
— (1998). Enseigner l'oral au cycle 3. En Dhers, D. et ál , Français, Concours de Professeur des Écoles, Tome 1 (227-249). Paris: Hatier.

Grandaty, M. (1998). Élaboration a plusieurs d'une conduite d'explication en sciences, au cycle 2, Repères. L'oral pour apprendre, (17): 109-125.

Grupo EVA (1996). De l'évaluation à la réécriture. Paris: Hachette Education.

Halliday, M. A. K. (1985). Spoken and written language. Oxford: Oxford University Press.

Halte, J. F. (Ed.) (1993). Inter-actions. Université de Metz.

Huberman, M. (1986). Un nouveau modèle pour le développement professionnel des enseignants. Révue Française de Pédagogie, (75): 5-15.

Lahire, B. (1993). Culture écrite et inégalités scolaires. Sociologie de I'"échec scolaire" a l'école primaire. Lyon: Presses Universitaires de Lyon.

Le Collège des années 2000. Suplemento del B.O. No 23. 10 de junio de 1999: 24-25.

Le Cunff, C. \& Groupe Oral-Créteil Patrick Jourdain (1999). Le rôle de l'enseignant 2. L'évaluation. Enseigner l'oral a l'école primaire. Paris: Hachette Éducation.

Maquaire, M. (1998). Enseigner l'oral. En Maquaire, M. (Ed.), Vers la maitrise des discours (47-60). CRDP de Bretagne.

Morel, M. A. \& Danon-Boileau, L. (1998). Grammaire de I'intonation. L'exemple du français. Bibliothèque des faits de langue. Paris: Ophrys.

Nonnon, E. (1992). L'oral: un fantôme omniprésent, ou un cadavre encombrant pour la didactique? Innovations (23-24): 9-22.

- (1997). Quels outils se donner pour lire la dynamique des interactions et le travail sur les contenus de discours? Enjeux, (39/40): 12-49.

Revista Recherches, AFEF LILLE, $\mathrm{N}^{\circ}$ 9, Argumenter (noviembre de 1998) y $\mathrm{N}^{\circ} 14$, Situations d'argumentation (septiembre de 1991).

Sacks, H., Schegoff, A. \& Jefferson, G. (1974). A simplest systematic for the organization of turn-taking for conversation. Language, 50(4): 696-735. 